\title{
Homo narrans : le rôle de la narration dans l'émergence du langage
}

\author{
Bernard Victorri
}

A dog cannot relate his autobiography; however eloquently he may bark, he cannot tell you that his parents were honest though poor.

Russel (1948:74)

\section{Introduction}

La plupart des chercheurs qui travaillent aujourd'hui au problème de l'origine du langage s'accordent à penser que le système de communication des hominidés se serait développé par étapes. En effet, on sait aujourd'hui ${ }^{1}$ que le processus d'hominisation a duré six à huit millions d'années et qu'il s'est déroulé luimême par étapes, avec notamment deux stades essentiels (du moins pour notre propos) : l'apparition de l'Homo erectus, il y a plus d'un million d'années, et la naissance de notre propre espèce, l'Homo sapiens «moderne », il y a quelque cent mille ans. Homo erectus a représenté un incontestable succès évolutif : premier hominidé à sortir d'Afrique, il a conquis tout l'Ancien Monde, et il a connu un développement continu de ses facultés cognitives et de sa technologie, conduisant ainsi à la naissance des différentes lignées d'Homo sapiens «archaïques » (y compris l'homme de Neandertal), qui maîtrisaient le feu, la chasse aux gros mammifères, la construction d'habitats, etc. Quant à notre espèce, issue de l'un de ces groupes d'Homo sapiens archaïques en Afrique ou au Moyen Orient, elle s'est à son tour dispersée sur toute la planète, supplantant les autres descendants d'Homo erectus, et elle est responsable de «l'explosion symbolique », il y a quelque quarante mille ans (cf. Tattersall 1998), premier signe indubitable de la maîtrise de l'intégralité des capacités cognitives humaines. Il y a donc de bonnes raisons de supposer qu'à ces deux étapes correspondent aussi des innovations dans le système de communication des espèces concernées.

A la suite de Bickerton (1990) et de bien d'autres, nous ferons donc l'hypothèse que les hominidés qui nous ont précédés (Homo erectus et ses descendants: Néandertaliens et autres Homo sapiens " archaïques ») étaient déjà dotés d'un "protolangage », système de communication plus rudimentaire que le langage proprement dit. Selon cette hypothèse le protolangage ne possédait pas un certain nombre de propriétés syntaxiques et sémantiques des langues humaines; il était néanmoins bien adapté à des actes simples de communication portant sur la réalité sensible immédiate (hic et nunc). Pour ces actes, des mécanismes pragmatiques simples peuvent aisément suppléer l'absence de ces propriétés plus complexes de nos langues.

Nous n'aborderons pas ici le problème de l'émergence du protolangage lui-même. Plusieurs auteurs (entre autres Dunbar, 1996, et Dessalles, 1998, 2000) ont récemment avancé des pistes intéressantes pour résoudre ce problème, plus délicat qu'il n'y paraît à première vue. Pour le néo-darwinisme, il constitue en effet un paradoxe, étant donné le comportement «machiavélique » qui caractériserait les sociétés de primates (cf. Byrne \& Whiten, 1988) ${ }^{2}$.

Nous ne prendrons pas partie non plus dans les controverses, en partie liées au problème précédent, sur la nature exacte et les fonctions de ce protolangage. Certains, comme Bickerton (1990, 1995), ont défendu l'idée qu'il était avant tout destiné à transmettre de l'information factuelle et qu'à ce titre, il était essentiellement constitué par un lexique, lequel se serait enrichi continûment en corrélation avec

\footnotetext{
${ }^{1}$ Pour une présentation des théories récentes de l'hominisation, s'appuyant notamment sur la génétique des populations, voir par exemple Lewin 1989, Cavalli-Sforza 1996, Tatterstall 1998 ou de Lumley 1998.

${ }^{2}$ En quelques mots, il s'agit de comprendre comment la sélection naturelle a pu aboutir à l'émergence d'un comportement « altruiste » de diffusion d'informations pertinentes dans des groupes où la finalité première de la communication semble être la «manipulation » du congénère (impliquant au contraire la rétention d'information et la simulation).
} 
l'augmentation de la taille du cerveau qui caractérise l'évolution d'Homo erectus. D'autres au contraire, comme Donald (1991), privilégient une fonction de cohésion sociale et font valoir qu'un véritable lexique n'a pu apparaître qu'après l'acquisition d'une capacité symbolique qui nous serait propre. En fait, la seule hypothèse dont nous aurons besoin ici n'est pas sujette à controverse : il s'agit de l'incapacité du protolangage à permettre l'évocation d'événements passés ou imaginaires, qui ne font pas l'objet de l'attention immédiate des interlocuteurs.

Nous nous intéresserons dans cet article à la naissance du langage proprement dit, tel que nous le connaissons au travers des milliers de langues parlées de nos jours. Cette dernière étape dans l'évolution du système de communication des hominidés serait donc le fait de notre propre espèce. En permettant le développement du monde symbolico-culturel dans lequel nous vivons, l'apparition du langage a profondément transformé la nature et le rythme de l'évolution, conférant à l'Homme la place singulière qu'il occupe dans le règne animal. Il s'agit de comprendre comment un processus évolutionniste a pu produire un tel changement qui a permis à notre espèce d'échapper en grande partie aux contraintes de l'évolution néo-darwinienne pour s'inscrire dans une logique évolutive différente, dont le centre organisateur s'est déplacé du biologique vers le social et le symbolique.

Pour expliquer le passage du protolangage au langage proprement dit, nous développerons l'idée de l'émergence d'une nouvelle fonction de communication qui pourrait avoir été le moteur de l'acquisition des propriétés spécifiques à nos langues. La thèse proposée ici appartient donc au courant fonctionnaliste des théories de l'émergence du langage, comme celles de Donald (1991) ou de Knight (1998) par exemple. Elle se distingue des approches de Pinker (1994) ou de Bickerton (1998), qui sont, à l'inverse, d'inspiration structuraliste et cognitiviste. La nouvelle fonction qui aurait déclenché le processus serait la fonction narrative. La narration constitue, on le sait, l'une des plus importantes utilisations du langage dans toutes les sociétés humaines connues, des sociétés de chasseurs-cueilleurs aux sociétés les plus technologiquement avancées, et cette fonction était clairement hors de portée d'un protolangage, quelle que soit l'idée que l'on s'en fasse. Comme nous le verrons, la fonction narrative permet d'expliquer l'essentiel des caractéristiques du langage humain, et son émergence pourrait avoir constitué le facteur décisif expliquant la différence de destinée entre nos ancêtres directs et leurs proches cousins comme les Néandertaliens.

\section{Les spécificités du langage humain}

Dans les recherches sur l'origine du langage, l'une des méthodes les plus importantes consiste à examiner les propriétés qui sont à la fois communes à toutes les langues connues (les universaux) et absentes des autres systèmes de communication (les spécificités du langage humain). On peut en effet penser que ces propriétés sont liées à des fonctions fondamentales du langage humain, et qu'elles ont été présentes dès son apparition. Beaucoup d'auteurs utilisant cette approche se sont concentrés sur la syntaxe, négligeant la sémantique, notamment la sémantique grammaticale. Pinker et Bloom (1990) par exemple ont mis l'accent sur des propriétés syntaxiques comme la récursivité ou l'enchâssement, qui rapprochent les langues humaines des langues artificielles que sont les formalismes logiques et informatiques. Ces propriétés ne sont donc pas spécifiques au langage humain (même si elles s'y expriment de manière particulière). Il vaut donc mieux se tourner vers les propriétés sémantiques des langues, pour lesquelles, comme nous allons le voir, le langage humain apparaît obéir à des contraintes spécifiques qui l'éloigne au contraire des langages formels.

L'une de ces propriétés les plus frappantes concerne l'expression de la temporalité. Contrairement aux langages logiques, les énoncés de base d'une langue sont intrinsèquement dynamiques. Les phrases les plus élémentaires des langues ne sont pas les célèbres Socrate est mortel et Tous les hommes sont mortels, mais bien plutôt du genre Il se met à pleuvoir, Le bébé pleure encore ou Je quitte Paris demain matin. Autrement dit, les langues permettent de parler de situations dynamiques inscrites dans le temps de manière simple et directe, ce qui n'est pas le cas des langages formels, comme le montre la complexité des logiques temporelles (cf. par exemple Audureau, Enjalbert et Farinas del Cerro, 1990,ou Bestougeff et Ligozat, 1989). De plus, pour l'expression de cette dynamique temporelle, les langues possèdent une 
catégorie sémantique qui n'existe tout simplement pas dans les logiques temporelles, si complexes soientelles : il s'agit de l'aspect. Comme on le sait, l'aspect occupe un place primordiale dans l'expression langagière : toutes les langues possèdent des marqueurs aspectuels. Au contraire, l'expression du temps proprement dit (présent, passé, futur) n'est pas grammaticalisée dans de nombreuses langues, comme le chinois par exemple ( $\mathrm{Li}$ et Thompson 1989). Quelle est la fonction de l'aspect ? Ce n'est pas la transmission d'information factuelle, pour laquelle seule l'information temporelle compte. En revanche, l'aspect joue un rôle primordial dans la narration : il permet de présenter les événements au cours de leur déroulement, d'adopter le point de vue de tel ou tel personnage à un instant donné, de plonger les interlocuteurs au cœur de l'action ou au contraire de prendre du recul par rapport à ce qui est évoqué. De plus les marqueurs purement temporels, si tant est qu'il en existe, se trouvent également mieux décrits en terme de mécanismes narratifs. Le temps présent, on le sait, peut servir aussi bien à évoquer des évènements passés que futurs. Comme le souligne Turner (1996:149-154), le temps verbal dépend du choix d'un point de vue par le narrateur. Rien ne lui impose de s'ancrer sur l'instant de l'énonciation. De manière plus approfondie, Gosselin (1996) et Klein (1994) ont proposé des modèles complets de la sémantique aspectuo-temporelle de plusieurs langues; ces modèles font jouer un rôle essentiel à une «fenêtre temporelle » (appelée intervalle de référence ou d'assertion) qui est ouverte par le locuteur à sa guise, donnant ainsi au narrateur la même facilité pour se déplacer dans le temps que celle dont dispose un metteur en scène.

On peut faire des observations similaires en ce qui concerne d'autres grands domaines de la sémantique grammaticale : expression des modalités, de l'agentivité, de la détermination, des relations spatiales... Si les systèmes de marqueurs impliqués peuvent sembler imparfaits, sinon incohérents, à un logicien ou à un spécialiste de la théorie de l'information, ils se révèlent comme des outils parfaits à la disposition d'un narrateur qui veut présenter à sa façon des scènes passées ou imaginaires, sans relation directe avec la situation présente.

Prenons l'exemple des modalités. On sait que les verbes modaux, comme pouvoir et devoir, possèdent plusieurs sens, notamment un sens déontique et un sens épistémique. D'un point de vue logique, cela paraît étrange. Une phrase comme Il peut fumer dans sa chambre peut avoir deux sens très différents quant à sa valeur de vérité : a-t-il le droit de fumer dans sa chambre, ou se peut-il qu'il y fume effectivement ? Cette situation est très répandue dans les langues, et bien au delà de la famille indoeuropéenne. Kronning (1996:92-93), par exemple, cite une vingtaine de langues, d'une dizaine de familles différentes (sino-tibétaine, austronésienne, etc.) dans lesquelles existe un équivalent de devoir muni de ces deux interprétations déontique et épistémique. Si l'on s'en tient à une approche strictement informationnelle, ce phénomène reste très difficile à comprendre : l'effort nécessaire pour désambiguïser ces marqueurs, pourtant fréquents, constitue une charge cognitive dont on ne voit pas la raison d'être dans l'économie des échanges d'information. Au contraire, si l'on admet que les unités linguistiques sont avant tout des outils de construction de scènes narratives, ce problème n'en est plus un. En effet, la sémantique des verbes modaux peut être complètement décrite en terme d'accès (Sweetser 1990, Culioli 1999). Dans cette optique les marqueurs modaux servent à indiquer le statut de la nouvelle scène évoquée par l'énoncé qui les porte par rapport aux scènes déjà construites (voir aussi Fauconnier 1997). L'accès à cette nouvelle scène peut être nécessaire (il n'existe alors pas de chemin conduisant à une situation différente), simplement possible (au moins deux chemins divergents existent), ou impossible (il n'y a pas de chemin vers la situation évoquée). Ce sont ces distinctions qui sont les plus pertinentes du point de vue du processus narratif où chaque nouvelle scène est construite à partir de ce qui a déjà été mis en place dans l'espace intersubjectif de la parole. Ce sont elles qui sont les premières à être grammaticalisées. D'autres distinctions, comme la différence entre épistémique et déontique, peuvent être exprimées dans n'importe quelle langue, mais leur moins grande grammaticalisation prouve qu'elles sont moins fondamentales du point de vue de l'activité langagière.

Prenons un autre exemple : les démonstratifs. Dans les langues qui en possèdent, les démonstratifs ont deux usages distincts : anaphorique et déictique. Cette ambivalence a été abondamment discutée (pour une synthèse, voir Kleiber 1991). Les sémanticiens cherchent à en comprendre les bases cognitives. En 
termes de transmission d'information factuelle, ceci est à nouveau problématique : désigner une entité présente dans l'environnement et ré-évoquer quelque chose ou quelqu'un que l'on vient d'évoquer dans le discours constituent deux opérations nettement distinctes au plan informationnel. En revanche, si l'on accepte l'idée que la fonction essentielle du langage consiste à conférer une présence phénoménologique aux entités et événements évoqués par le discours, ce mécanisme se révèle d'une efficacité remarquable : utiliser les marqueurs déictiques pour ré-évoquer des entités discursives revient à les doter de la même présence indiscutable que les entités qui se trouvent devant soi. L'outillage linguistique apparaît davantage conçu pour réaliser cette «simulation de perception » (Gosselin sous presse) que pour transmettre simplement de l'information.

Enfin la syntaxe elle-même peut être abordée dans ce cadre. Turner (1996:143-148) montre par exemple que les constructions grammaticales à rôles actanciels peuvent être interprétées comme des projections «d'histoires abstraites élémentaires » (basic abstract stories). Il montre également que les enchâssements et la récursivité correspondent à des projections «d'emboîtements d'histoires » (story nesting), par insertion et combinaison de ces histoires élémentaires. Comme il le note (Turner 1996:161), cette conception de la syntaxe vue comme «système dynamique de constructions grammaticales » s'inscrit parfaitement dans un certain nombre de modèles de linguistique cognitive (Goldberg 1995, Talmy 1988). Un autre ensemble de phénomènes syntaxiques, traités dans les cadres fonctionnalistes (Givón 1995, Halliday 1994), est peut-être encore plus pertinent pour notre discussion. Il s'agit de ce que Lambrecht (1994) appelle la «structure informationnelle ». Cela concerne tous les outils grammaticaux et procédés syntaxiques servant à introduire l'information nouvelle (ou rhématique) en l'ancrant dans le contexte discursif $^{3}$. Ces mécanismes sont essentiels à la narration, puisque le succès d'un récit dépend de manière cruciale de la capacité à évoquer de nouveaux personnages ou événements sur l'unique base de ce qui a déjà été mis en scène. Certains de ces mécanismes paraissent d'ailleurs conçus entièrement à des fins narratives, comme les divers systèmes de «poursuite de la référence » (reference-tracking systems), que l'on trouve dans de nombreuses langues (cf. Van Valin et LaPolla 1997:285-290).

Ainsi la plupart des spécificités des langues humaines, qui les différencient tant des autres systèmes de communication animale que des langages logiques et autres langages formels, semblent directement liées à la fonction narrative du langage. On peut donc avancer l'hypothèse que la fonction narrative serait à la racine même de l'émergence du langage. C'est cette hypothèse que développe Mark Turner (1996, chap. 8). Il suppose que «l'imagination narrative» (narrative imagining) a d'abord été une capacité cognitive individuelle résultant de l'augmentation de l'intelligence et de la mémoire des hominidés, qui leur aurait permis d'évoquer dans leur propre esprit le déroulement d'événements passés ou imaginaires. Dans un deuxième temps, cette structure aurait été projetée dans le système de communication de ces hominidés, le transformant alors en langage humain. Ainsi Turner s'oppose directement aux thèses de Chomsky (1995), Pinker et Bloom (1990), ainsi que Bickerton (1984) qui soutiennent que l'acquisition génétique d'un organe du langage inné a permis de développer les fonctionnalités du langage. C'est, à l'inverse, le besoin fonctionnel de raconter des histoires qui aurait forgé les propriétés du langage. Comme le dit Turner (1996:162) :

«Perhaps the main argument that grammar must arise in the individual human being exclusively from some special-purpose device, genetically coded and neurobiologically expressed, is that grammar is too arbitrary, subtle and quirky to arise otherwise. But if the influence on language acquisition is not only the language an infant hears, but also all of narrative imagining, including all of the systems from which narrative imagining recruits, there is plausibly an overabundance of sources of subtleties and quirks without conjecturing a special device to introduce them ».

\section{Le problème de l'extinction presque totale des Homo sapiens archaïques}

Ainsi l'émergence d'une nouvelle fonction, la fonction narrative, pourrait expliquer la transformation du protolangage en langage : le besoin d'évoquer des événements passés ou imaginaires aurait conduit à

\footnotetext{
${ }^{3}$ Voir aussi les notions de «profile» et «landmark» chez Langacker, 1986, et la notion de repérage dans la théorie énonciative de Culioli, 1990, 1999).
} 
améliorer progressivement le système de communication de nos ancêtres en le dotant de toutes les propriétés syntaxiques et sémantiques des langues humaines. Mais pourquoi et comment ce besoin auraitil émergé dans une population d'Homo sapiens archaïque ? Pour répondre à cette question, il nous faut examiner plus en détail les problèmes que pose la dernière étape de l'hominisation, au cours de laquelle notre espèce est apparu.

Comme nous l'avons dit, les descendants des Homo erectus, qui avaient évolué localement dans tout l'Ancien Monde pour aboutir aux différents groupes d'Homo sapiens archaïques, ont pratiquement tous disparu par la suite (il y a quelque trente mille ans pour les derniers Néandertaliens). Seul l'un de ces groupes, en Afrique de l'Est ou au Moyen Orient, a connu un sort différent en donnant naissance à notre espèce avec le succès que l'on sait. Il y a donc deux problèmes liés à résoudre : pourquoi presque tous les groupes d'Homo sapiens archaïques ont disparu et qu'est-ce qui a permis à notre espèce d'éviter cette extinction.

La plupart des auteurs s'accordent à dire que l'extinction des autres Homo sapiens archaïques, et notamment des Néandertaliens, reste une véritable énigme. Trois types d'explications ont été avancées : le climat, des épidémies ou la compétition avec notre propre espèce. Mais ces explications sont loin d'être satisfaisantes.

Les changements climatiques sont invoqués notamment par Reichholf (1990). Celui-ci explique que ces changements, à la fin de la dernière glaciation, ont provoqué la disparition en Europe de quelques espèces de gros gibier (mammouths, rhinocéros, ...). Cela aurait été fatal aux Néandertaliens, parce qu'ils auraient dépendu trop fortement de ce gibier pour leur alimentation. Ce raisonnement est difficile à accepter dans la mesure où toute l'évolution depuis Homo erectus s'est centrée sur le développement d'aptitudes cognitives permettant de s'adapter à toute sorte d'environnements. Il serait surprenant que des descendants de cette espèce, encore mieux armés sur le plan cognitif (l'homme de Neandertal avait même un cerveau d'une taille légèrement supérieure à la nôtre), n'aient pas été capables de changer leur mode de vie, et en particulier leur comportement alimentaire, au fur et à mesure des changements climatiques, alors qu'ils avaient maîtrisé le feu, la chasse et la cueillette en groupe, la construction d'habitats, etc.

Les épidémies ne constituent pas non plus une explication convaincante. Les Homo sapiens archaïques étaient dispersés dans tout l'Ancien Monde, et il serait assez surprenant que des épidémies les aient frappés partout. Qui plus est, une épidémie peut décimer une population, mais rarement l'éliminer complètement, à moins que cette population ne soit déjà très faible numériquement. Si l'on accepte cette dernière hypothèse, il reste donc à comprendre pourquoi, après l'immense succès évolutif $d$ 'Homo erectus, qui s'est concrétisé par la colonisation de tant de territoires, ces descendants étaient devenus si peu prospères au point d'être menacées de disparition par des épidémies.

Il en est de même pour la dernière des raisons souvent invoquées, la compétition avec notre propre espèce, avec laquelle les Néandertaliens notamment ont coexisté un certain temps. Il n'y aurait eu, comme le dit Donald (1991), de la place que pour une espèce dans la niche écologique occupée par Homo, et c'est notre espèce qui l'aurait emporté sur les espèces voisines. A nouveau, cela ne saurait constituer le fin mot de l'histoire. A moins que nos ancêtres n'aient systématiquement planifié et réalisé l'éradication complète de leur lointains cousins (et cela sonne trop moderne pour être plausible, surtout qu'ils ne semblaient pas disposer alors d'une supériorité technologique importante dans leur armement), comment expliquer que ceux-ci aient complètement disparu dans cette compétition au lieu d'être simplement repoussés plus loin (la Terre n'était pas si petite, à l'époque tout au moins !), dans des régions peut-être moins hospitalières, mais où ces êtres si intelligents et adaptables auraient pu survivre ? Il faut là encore supposer qu'ils aient été déjà fragilisés pour d'autres raisons. Ainsi, ce qu'il faut découvrir, ce sont les causes de cette faiblesse intrinsèque qui les a rendu si vulnérables, au point de ne pas pouvoir résister à des agressions externes, qu'elles proviennent d'un changement climatique, d'un quelconque virus, ou de représentants de notre espèce.

Il faut donc se pencher sur des causes endogènes qui pourraient expliquer l'extinction des Homo sapiens archaïques. La thèse que nous défendrons ici est celle d'un phénomène de dérégulation sociale, dû précisément à l'augmentation de l'intelligence individuelle de ces hominidés. Cette thèse peut paraître 
paradoxale : on a du mal à concevoir l'hominisation, et en particulier l'accroissement constant des facultés cognitives, autrement que comme un progrès. Mais, comme nous allons le voir, cela a pu conduire, au contraire, à une véritable impasse évolutive (Victorri 1999, 2000).

Toute organisation sociale, quelle soit animale ou humaine, nécessite le strict respect par les membres du groupe d'un certain nombre de règles comportementales qui peuvent être contraires à leur intérêt individuel à court terme, mais qui sont vitales pour la viabilité du groupe. Dans le monde animal (Lorenz 1970, 1977), ces comportements dangereux pour la survie de l'espèce sont inhibés par des mécanismes dits «instinctifs ». En particulier, chez les mammifères sociaux, ce sont ces mécanismes, profondément ancrés dans les couches les plus primitives du cerveau, qui régulent les comportements agressifs : les combats au sein du groupe, pourtant fréquents, ne se soldent jamais, ou très exceptionnellement, par la mise à mort du vaincu ${ }^{4}$. Chez l'homme la situation est fort différente : la régulation sociale ne s'effectue pas au niveau biologique, mais au niveau socioculturel. Les comportements à prohiber, tels que tuer son frère ou son père par exemple, font l'objet d'interdits explicites dans toutes les sociétés humaines. De cette différence, capitale, on peut tirer plusieurs enseignements sur l'évolution des hominidés.

Primo, cela signifie que, chez l'homme, le contrôle biologique de ces comportements dangereux pour l'espèce est inexistant, ou, tout au moins, considérablement affaibli. En effet, si ce n'était pas le cas, leur interdiction explicite n'aurait pas lieu d'être : c'est précisément parce nous sommes biologiquement capables de commettre de tels actes (les exemples ne manquent pas...) qu'ils sont culturellement prohibés. Secundo, cela implique qu'il a existé une période où les inhibitions instinctives étaient déjà sérieusement affaiblies alors que les règles sociales qui les remplacent aujourd'hui n'étaient pas encore en place. Il est en effet peu vraisemblable, toujours pour les même raisons, que l'établissement de ces règles ait précédé la perte des réactions instinctives.

Ce processus peut s'expliquer dans le cadre de l'évolution biologique des hominidés. L'hominisation s'est caractérisé par un développement considérable du néocortex (notamment des aires frontales, cf., par exemple, Ackerman 1992). Ces zones contrôlent les centres sous-corticaux responsables des réactions instinctives chez les mammifères. Ainsi l'accroissement des capacités cognitives des hominidés a conduit progressivement à la maîtrise des comportements instinctifs, auxquels se sont substitués des comportements plus adaptables et plus «réfléchis ». Autrement dit, le développement de l'intelligence individuelle a eu pour corollaire la perte des réactions instinctives, y compris, in fine, de celles qui étaient les plus solidement établies, parce que vitales pour la survie de l'espèce, comme celles qui régulaient l'agressivité au sein du groupe.

On conçoit donc qu'un même processus ait pu être responsable à la fois du succès évolutif des Homo erectus et des difficultés qu'ont subies leurs descendants, les Homo sapiens archaïques. La pression évolutive en faveur d'un développement toujours plus important du néocortex a d'abord eu un effet très positif, en dotant les premiers Homo de capacités nouvelles qui leur ont permis d'inventer de nouveaux modes de vie et de prospérer dans tous les territoires qu'ils ont conquis. Mais à un certain stade, cette même pression évolutive a abouti à une domination presque totale du néocortex qui a mis en danger l'espèce en affaiblissant les contraintes instinctives qui régulaient la vie sociale.

L'éthologie animale, et plus particulièrement les nombreuses études sur le comportement social des singes anthropoïdes, comme par exemple celles de F. de Waal (1995) sur les chimpanzés, peuvent nous aider à concevoir le type de crises auxquelles ont dû être confrontés les différents groupes d'Homo sapiens archaïques.

On sait que la vie sociale des groupes de chimpanzés est ponctuée de crises, plus ou moins violentes, qui mettent aux prises des mâles dominants en lutte pour le leadership du groupe. Généralement, ces conflits couvent un certain temps, chacun des rivaux cherchant à constituer une coalition qui le soutienne, et cela aboutit à une confrontation directe entre les rivaux, soutenus par leurs alliés. A l'issue du combat, qui est

\footnotetext{
${ }^{4}$ Ces observations concernent uniquement les comportements «meurtriers » au sein d'un même groupe, qui menacent directement l'organisation sociale du groupe. En revanche, dans les conflits inter-groupes, ces inhibitions ne sont plus de mise : on a ainsi pu observer de véritables «guerres » entre groupes de chimpanzés, avec des tueries systématiques (cf. Goodall 1986).
} 
suffisamment régulé pour ne pas mettre en danger la vie des protagonistes, le vaincu marque sa soumission, et le vainqueur peut exercer son pouvoir sur le groupe sans contestation jusqu'à la prochaine crise. C'est ainsi que se maintient et se renouvelle une hiérarchie de dominance assez complexe dont le rôle est essentiel dans tous les aspects de la vie sociale du groupe (comportements alimentaires, sexuels, protection des jeunes, etc. : cf. de Waal, 1995).

On peut raisonnablement penser que l'organisation sociale des hominidés était régie par des processus analogues. Mais dans les groupes d'Homo sapiens archaïques, moins soumis aux régulations instinctives, le scénario peut prendre une autre tournure. Les deux protagonistes d'un conflit de ce type ont l'intelligence suffisante pour projeter de tuer leur rival, en le prenant par surprise et en utilisant leurs armes les plus efficaces. Chacun peut prêter à l'autre l'intention de nourrir un tel dessein, ce qui ne peut que le pousser à agir le premier, pour éliminer ce danger. Ce sont des comportements intelligents, que l'on est en droit d'attribuer à des animaux possédant de grandes capacités cognitives et de faibles inhibitions instinctives. Notons aussi que tout le groupe est concerné : plusieurs membres du groupe peuvent être liés à l'un des rivaux par des relations de coalition, ou en être suffisamment proches affectivement pour se sentir impliqués dans le conflit, ce qui fait que le premier meurtre risque d'en engendrer d'autres. Ainsi, ce type de situation peut provoquer une crise majeure, déclenchant une violence incontrôlable désastreuse pour la survie du groupe. De toute manière, même si une telle réaction en chaîne est évitée, la perte d'un mâle au faîte de sa puissance constitue du point de vue évolutif un sérieux handicap (pour la chasse, la protection contre les agressions extérieures, etc.).

On peut imaginer d'autres situations de crise, tout aussi désastreuses. Après une longue période de chasse infructueuse, durant ces longs hivers où le gibier devait être la seule ressource alimentaire, les mâles dominants pouvaient penser que le seul moyen de survivre consistait à manger leurs propres petits : ils avaient besoin de recouvrer leurs forces, s'ils voulaient avoir une chance de rapporter du gibier les jours suivants, et les bébés allaient mourir de toute façon, puisque leurs mères ne pouvaient plus les nourrir. Là encore, il s'agit d'un comportement rationnel, plausible pour des individus capables de surmonter leur répulsion instinctive à de tels actes. Comme chacun était tenaillé par la même faim et capable des mêmes raisonnements, c'est tout le groupe qui pouvait anticiper ce comportement, y compris les mères, sans doute mues par des réactions instinctives protectrices mieux ancrées qui devaient les pousser à défendre leur propre progéniture par tous les moyens. Le groupe courait donc le risque d'un déchaînement de violence non maîtrisable, et de toute façon subissait avec la mort des petits une perte démographique irrémédiable, que des chasses fructueuses par la suite ne pouvaient compenser.

En présentant ces «scénarios », nous n'avons pas, on s'en doute, la moindre prétention à une description « réaliste » d'éléments de la vie sociale des Homo sapiens archaïques. Il s'agit simplement d'illustrer de façon concrète un principe général : le développement de l'intelligence individuelle peut engendre des comportements anti-sociaux, néfastes pour la survie de l'espèce. En l'absence de contraintes, biologiques ou sociales, capables d'en contrecarrer les effets, la branche Homo a donc bien pu se trouver, pendant toute une période, soumise à des crises sociales qui peuvent expliquer cette faiblesse intrinsèque que nous avons qualifiée d'impasse évolutive.

\section{L'émergence de la fonction narrative}

Si l'on accepte que la répétition de ce type de crises a constitué la cause principale de la fragilisation des groupes d'Homo sapiens archaïques, on en déduit que notre espèce a pu échapper à l'extinction parce qu'elle s'est dotée d'un mécanisme qui a permis d'éviter ces crises. C'est l'instauration de lois sociales, fondées sur des interdits explicites, qui a rétabli l'équilibre, et cela n'a pu avoir lieu que grâce à un progrès de la communication langagière. Le langage humain aurait donc émergé au cours de ce processus. On sait que tous les mythes et religions fondent les interdits sur des récits mettant en scène des personnages sacrés (ancêtres ou dieux) qui violent précisément ces interdits. En fait, les deux exemples que nous avons choisis, un frère qui tue son propre frère (ou son père) et un père qui dévore ses propres enfants, font partie des figures mythiques les plus répandues dans le monde, et les interdits 
correspondants ont un statut d'universaux pour toute l'humanité ${ }^{5}$. La fonction narrative du langage joue donc un rôle capital dans l'expression des lois sociales qui suppléent, chez l'homme, aux inhibitions instinctives.

Notre thèse peut alors se résumer de la manière suivante. Pour échapper aux crises récurrentes qui déréglaient l'organisation sociale, nos ancêtres ont inventé un mode inédit d'expression au sein du groupe : la narration. C'est en évoquant par la parole les crises passées qu'ils ont réussi à empêcher qu'elles se renouvellent. Le langage humain s'est forgé progressivement au cours de ce processus, pour répondre aux besoins nouveaux créés par la fonction narrative, et son premier usage a consisté à établir les lois fondatrices qui régissent l'organisation sociale de tous les groupes humains.

Pour illustrer concrètement comment un tel processus a pu se mettre en place, revenons à nos «scénarios ». Remarquons d'abord que les crises devaient susciter des conflits intérieurs chez les protagonistes de ces drames, déchirés entre leur répugnance instinctive résiduelle à commettre de tels actes, et leurs désirs, s'appuyant sur leur intelligence, de le faire malgré tout. Et tous les membres du groupe, à des degrés divers, devaient ressentir le même conflit. On peut aussi supposer que beaucoup d'entre eux se souvenaient des crises précédentes et des désastres qui s'en étaient suivis. Si un de ces individus se révélait alors capable d'évoquer ce passé, par la voix et les gestes, il avait une chance de capter l'attention du groupe et de stopper la catastrophe imminente.

Prenons l'exemple du conflit entre deux mâles en rivalité pour le leadership du groupe. Supposons donc qu'à un instant critique, après un nouvel accrochage entre les deux rivaux, un membre âgé du groupe se lance dans cette entreprise de remémoration collective. On peut se demander comment il aurait pu s'y prendre, en l'absence d'un langage capable de faire référence à des événements « hors-contexte », qui ne sont pas au centre des préoccupations des interlocuteurs. C'est bien sûr « la » question que doit résoudre tout scénario de l'origine du langage, question qui ne se pose pas pour le protolangage, qui reste cantonné à l'ici et au maintenant, et pour lequel la référence de tout nouveau signe peut être devinée à partir de la situation, permettant à ce signe d'acquérir progressivement un sens conventionnel. Notons que dans notre exemple, le but est au contraire de détourner l'attention de la situation présente pour pouvoir la juguler. Néanmoins, il faut aussi remarquer que les conditions de cette entreprise sont optimales au sens où beaucoup de membres du groupe doivent aussi avoir en mémoire ces événements passés, dont le souvenir est ravivé par l'appréhension de ce qui va se passer. Qui plus est, ils savent que ce vieil individu n'est pas directement impliqué dans le présent conflit, et qu'il a lui aussi peur de ses conséquences. Ils peuvent donc anticiper que son intention est de l'arrêter par tous les moyens en sa possession.

Supposons alors que notre apprenti narrateur arrive à faire comprendre qu'il veut évoquer l'un des acteurs de cette crise passée, en utilisant quelque procédé mimétique: imitant l'une de ses particularités physiques, un animal qu'il aimait chasser, son cri favori, etc. Le succès d'une telle évocation était susceptible de produire une impression très forte sur tout le groupe : pour la première fois l'image d'un membre disparu du groupe apparaît devant eux, chacun prenant conscience que les autres partagent la même «vision ». Ce qui était cantonné dans des mémoires individuelles devient l'objet d'une attention collective, acquiert une présence intersubjective, «magique », qui frappe profondément les esprits. Le narrateur peut alors progresser tant bien que mal dans son proto-récit, faisant revivre les personnages devant le groupe subjugué, conscient de vivre collectivement une expérience tout à fait nouvelle. Cette conscience collective renforce la cohésion du groupe et lui confère un nouveau pouvoir. Raconter ce qui s'est déjà produit devient un moyen d'exprimer que cela risque d'arriver de nouveau et qu'il ne faut pas que cela se reproduise. Cette pression du groupe pouvait donc l'emporter, dans la mesure où les protagonistes étaient eux-mêmes ébranlés par cette évocation, et ne pouvaient plus ignorer que le conflit intérieur qui les déchirait était devenu l'affaire du groupe dans son ensemble.

\footnotetext{
${ }^{5}$ Ce n'est bien sûr pas le cas pour le meurtre d'un être humain en général, ni même pour la consommation de sa chair : encore récemment, le cannibalisme externe était une pratique sacrée dans diverses sociétés, comme les Tupi-Guarani (cf. Combès 1992) ou les Aztèques (Harris 1979). Les deux interdits dont nous parlons correspondent donc très précisément à des contraintes de survie du groupe, exactement comme les inhibitions instinctives qu'ils ont remplacées (cf. note 4).
} 
Bien sûr, les chances de réussite d'un tel artifice sont faibles. Mais, même en les supposant rares, les succès ont un impact immédiat sur les chances de survie du groupe. Cette innovation culturelle aurait donc pu se généraliser sur le long terme, puisqu'elle favorisait les groupes qui la pratiquaient, exactement comme, à une autre échelle, un trait génétique individuel se répand rapidement dans une population s'il est avantageux. Une étape importante dans ce processus peut avoir consisté à ritualiser le comportement narratif : au lieu d'attendre qu'une crise éclate, il est en effet plus efficace d'organiser des manifestations régulières pour évoquer ces scènes ancestrales et les actes à prohiber. Et c'est tout au long de cette évolution du comportement social que les techniques narratives auraient progressé, se seraient affinées et complexifiées, en devenant aussi de plus en plus conventionnelles. Le langage humain, avec toutes ses propriétés syntaxiques et sémantiques, serait l'aboutissement de ce processus : on rejoint ici la thèse de Turner que nous avons présentée plus haut.

Ainsi, les premiers usages de la fonction narrative auraient consisté à évoquer des crises passées pour interdire des comportements nuisibles à la survie de l'espèce, créant ainsi une organisation sociale totalement inédite dans le règne animal, conduisant à ce que Donald (1991) appelle le stade de la « culture mythique », et c'est ce qui aurait permis à notre espèce d'échapper aux dérégulations sociales ayant conduit à l'extinction des autres Homo sapiens archaïques.

De nombreux auteurs se sont penchés sur les mythes pour tenter d'y découvrir des indices de l'origine des cultures et des sociétés humaines. Chris Knight $(1991,1998)$ est certainement l'auteur qui a le plus travaillé dans cette direction, du moins en ce qui concerne la question précise de l'émergence du langage. Mais l'approche développée ici est beaucoup plus proche de la théorie de René Girard (1972, 1982), même si celle-ci n'aborde pas spécifiquement cette question. Pour expliquer pourquoi les mythes mettent systématiquement en scène des héros qui sont vénérés comme des dieux alors même qu'ils ont enfreint des interdits, Girard développe l'idée que ces personnages proviennent d'ancêtres ayant réellement existé qui auraient été tués par les leurs parce qu'ils avaient commis ces actes, ou, plus précisément parce qu'ils en avaient été accusés publiquement. Ils seraient devenus des dieux parce qu'en étant des boucs émissaires, ils avaient permis de ressouder la tribu en crise en détournant la violence sur eux-mêmes, et parce que du même coup, cela avait permis de fonder les interdits. La thèse proposée ici est légèrement différente et d'une certaine manière correspond à une lecture plus littérale du mythe : ce n'est pas de leur vivant, en tant que boucs émissaires, qu'ils auraient (bien involontairement) contribué à résoudre des crises, mais c'est l'évocation plus tard de leurs actes qui aurait évité que ces crises se renouvellent. Ce qui est commun à ces deux approches, c'est l'idée qu'un nouvel ordre social a pu naître de la nécessité d'échapper à des crises, en imposant des lois fondées sur la conscience d'appartenir à un même groupe pourvu d'une histoire collective qui fonde son identité.

On peut penser que le langage a d'abord été limité à ces narrations rituelles, et que pendant une longue période il s'est développé relativement indépendamment du protolangage utilitaire que devait posséder notre espèce, comme tous les Homo sapiens archaïques. Il n'y a en effet aucune raison pour que le protolangage, bien adapté à la communication quotidienne, ait été supplanté dans cette fonction, du moins dans un premier temps ${ }^{6}$. Le protolangage a pu bien sûr fournir au langage naissant une partie de son matériel. Notamment, si l'on accepte l'idée que le protolangage possédait un lexique riche, une partie de ce lexique a pu être empruntée par le langage, mais pour servir d'une toute autre manière. Par exemple, notre premier apprenti narrateur pourrait avoir utilisé des mots du protolangage pour évoquer le personnage qu'il voulait mettre en scène, en prononçant le nom d'une plante ou d'un fruit dont ce dernier était très friand, ou d'un animal auquel on pouvait le comparer. En d'autres termes, il aurait inventé les opérations fondamentales de la sémantique lexicale : la métonymie et la métaphore. La polysémie aurait été alors présente dès les débuts du langage, alors qu'elle aurait été inconnue dans le protolangage. Pour le protolangage, le mot pour 'lion' n'aurait signifié rien d'autre que l'animal, alors que dans le langage, il aurait pu désigner aussi bien un ancêtre fameux, le totem auquel appartient une partie de la tribu, et la

\footnotetext{
${ }^{6}$ Encore aujourd'hui, on trouve des traces de la spécificité de l'utilisation religieuse du langage, notamment dans les traditions orales, avec une langue sacrée uniquement destinée à cet usage, souvent dotée d'un pouvoir magique, voire dangereux, à manier avec précaution.
} 
puissance et le courage que l'on attribue à la fois à l'ancêtre et à l'animal. Il est intéressant de noter que la polysémie fait aussi partie de ces propriétés des langues qui les distinguent des autres systèmes de communication animale, aussi bien que des langages formels (Victorri et Fuchs 1996).

L'idée d'une coexistence prolongée entre protolangage et langage pourrait expliquer la longueur de l'intervalle de temps qui sépare, semble-t-il, l'apparition de notre espèce et «l'explosion symbolique » que l'on date de quelque quarante mille ans (cf. Tattersall 1998). Il aurait fallu attendre que le langage perde en partie son caractère sacré et sa puissance magique pour qu'il puisse graduellement envahir la sphère du profane. Ce lent processus aurait conduit à l'emprise de la pensée symbolique sur tous les aspects de la vie quotidienne, provoquant d'abord la révolution symbolique du Paléolithique Supérieur, et, plus généralement, alimentant ce désir profond de donner du sens à toute chose qui caractérise notre espèce : là encore, il est clair que la fonction narrative a joué un rôle de premier plan, puisque raconter une histoire pour rendre compte d'un phénomène naturel est, presque toujours, le premier pas vers des descriptions et des explications de nature plus scientifique.

\section{Conclusion}

L'hypothèse du rôle décisif de la fonction narrative dans l'émergence du langage présente donc un certain nombre d'avantages :

- Elle permet d'expliquer les propriétés syntaxiques et sémantiques spécifiques des langues, notamment celles que l'on ne retrouve pas dans les langages formels.

- Elle est compatible avec ce que l'on sait aujourd'hui des dernières étapes de l'histoire de l'hominisation.

- Elle permet de rendre compte de l'apparition d'un nouveau type d'organisation sociale, propre à notre espèce, dans lequel des lois socioculturelles remplacent, dans une large mesure, les contraintes sociobiologiques régissant le reste du monde animal.

Cette thèse est, bien sûr, hautement spéculative par bien des aspects. C'est le lot de toutes les théories actuelles sur l'origine du langage. En l'absence de preuves directes, on ne peut qu'échafauder des théories hypothétiques dont la cohérence repose sur les connaissances acquises par d'autres disciplines (linguistique, paléoanthropologie, etc.), et dont l'intérêt essentiel est de permettre des confrontations entre différentes conceptions de l'homme et du langage. C'est dans cet esprit que nous avons présenté cette thèse, qui fait de l'homme un Homo narrans, puisque ce n'est pas l'intelligence qui le distinguerait des autres espèces d'Homo sapiens qui l'ont précédé, mais la capacité à raconter sa propre histoire, source d'une nouvelle « sagesse » fondatrice des sociétés humaines.

\section{Références}

Ackerman S, Discovering the brain, National Academy Press, 1992.

Audureau E., Enjalbert P., Farinas del Cerro L., Logique temporelle; Sémantique et validation de programmes parallèles, Masson, 1990.

Bestougeff H., Ligozat G., Outils logiques pour le traitement du temps, Masson, 1989.

Bickerton D., The Language Bioprogram Hypothesis, Behavioral and Brain Sciences, 7:173:188, 1984.

Bickerton D., Language and SpeciesUniversity of Chicago Press, 1990.

Bickerton D., Language and Human Behavior, University of Washington, 1995.

Bickerton D., Catastrophic evolution: the case for a single step from protolanguage to full human language, in Hurford et al., 341-358, 1998.

Byrne R., Whiten A. (eds.), Machiavellian Intelligence. Social Expertise and the Evolution of Intellect in Monkeys, Apes and Humans, Clarendon Press, 1988.

Cavalli-Sforza L., Gènes, peuples \& langues, Odile Jacob, 1996.

Chomsky N., Language and Nature, Mind, 104:1-61, 1995.

Combès I., La tragédie cannibale chez les anciens Tupi-Guarani, P.U.F., 1992.

Culioli A., Pour une linguistique de l'énonciation, tome 1: Opérations et représentations, Ophrys, 1990.

Culioli A., Pour une linguistique de l'énonciation, tome 2: Formalisation et opérations de repérage, Ophrys, 1999. 
Dessalles J.L., Altruism, status and the origin of relevance, in Hurford et al., 130-147, 1998.

Dessalles J.L., Aux origines du langage - Une histoire naturelle de la parole, Hermès, 2000.

Donald M., Origins of Modern Mind: Three Stages in the Evolution of culture and Cognition, Harvard University Press, 1991.

Dunbar R., Grooming, Gossip and the Evolution of Language, Faber \& Faber, 1996.

Fauconnier G., Mappings in Thought and Language, Cambridge University Press, 1997.

Girard R., La violence et le sacré, Grasset, 1972.

Girard R., Le bouc émissaire, Grasset, 1982.

Givón T., Functionalism and Grammar, Benjamins, 1995.

Goldberg A., Constructions - a Construction Grammar Approach to Argument Structure, University of Chicago Press, 1995.

Gosselin L., Sémantique de la temporalité en français, Duculot, 1996.

Gosselin L., Temporalité et modalité, Presses de l'Université de Rouen, sous presse.

Goodall J., The Chimpanzees of Gombe: Patterns of Behavior, Belknap Press, 1986.

Halliday M.A.K., An introduction to Functional Grammar, Edward Arnold, 1994.

Harris M., Cannibales et monarques, essai sur l'origine des cultures, Flammarion, 1979.

Hurford J.R., Studdert-Kennedy M., Knight C. (eds.), Approaches to the Evolution of Language, Cambridge University Press, 1998.

Kleiber G., Anaphore-deixis : où en sommes-nous ?, L'information grammaticale, 51:3-18, 1991.

Klein W., Time in Language, Routledge, 1994.

Knight C., blood Relations: Menstruation and the Origins of Culture, Yale University Press, 1991.

Knight C., Ritual/speech coevolution: a solution to the problem of deception, in Hurford et al., 68-91, 1998.

Kronning H., Modalité, cognition et polysémie :sémantique du verbe modal devoir, Acta Universitatis Upsaliensis, 1996.

Lambrecht K, Information structure and sentence form, Cambridge University Press, 1994.

Langacker R., Foundations of Cognitive Grammar, Stanford University Press, 1986.

Lewin R., Human Evolution, Blackwell, $2^{\circ}$ ed., 1989.

Li C.N., Thompson S.A., Mandarin Chinese: A Functional Reference Grammar, University of California Press, 1989.

Lorenz K., Trois essais sur le comportement animal et humain, Seuil, 1970

Lorenz K., L'agression, Flammarion, 1977

de Lumley H., L'homme premier, Odile Jacob, 1998.

Pinker S., The Language Instinct, William Morrow and Co, 1994.

Pinker S., Bloom P., Natural language and natural selection, Behavioral and Brain Sciences, 13:707-727, 765-784, 1990.

Reichholf J., Das Rätsel der Menschwerdung, Deutscher Taschenbuch Verlag GMBH, 1990.

Russel B., Human knowledge - Its scope and limits, George Allen and Unwin Ltd., 1948.

Schuyt R., The Morphology of Slavic Verbal Aspect: A descriptive and Historical Study, Rodopi, 1990.

Sweetser E., From Etymology to Pragmatics, Cambridge University Press, 1990.

Talmy L., Force Dynamics in Language and Thought, Cognitive Science, 12:49-100, 1988.

Tattersall I., Becoming Human, Evolution and Human Uniqueness, Harcourt Brace and Co, 1998.

Turner M., The Literary Mind, Oxford University Press, 1996.

Van Valin R.D., LaPolla R.J., Syntax - Structure, meaning and function, Cambridge University Press, 1997.

Victorri B., La place de la fonction narrative dans l'émergence du langage et la structure des langues, T.L.E. (Théorie, Littérature, Enseignement), 17:23-38, 1999.

Victorri B. : The role of narration in the emergence of human language, Proceedings of the Third International Conference on the Evolution of Language, Paris, 2000.

Victorri B., Fuchs C., La polysémie : construction dynamique du sens, Hermès, 1996. 
de Waal F., La politique du chimpanzé, Odile Jacob, 1995. 